\title{
COX-2 inhibition in the endothelium induces glucose metabolism normalization and impairs tumor progression
}

\author{
LONGHUI ZHANG ${ }^{1 *}$, SUFEN LI $^{1 *}$, LAN LI $^{2}, \mathrm{ZHENGQIONG} \mathrm{CHEN}^{1}$ and YING YANG \\ ${ }^{1}$ Obstetrics and Gynecology Department, Xinqiao Hospital, Third Military Medical University, Chongqing 400037;
${ }^{2}$ Obstetrics and Gynecology Department, Chong Qing Health Center for Women and Children, Chongqing 400010, P.R. China
}

Received October 9, 2017; Accepted December 12, 2017

DOI: $10.3892 / \mathrm{mmr} .2017 .8270$

\begin{abstract}
Previous antitumor angiogenesis strategies have focused on targeting angiogenic signals. Encouragingly, the metabolism of tumor endothelial cells (TECs) has gained attention as a therapeutic target in recent years. There is consensus that, in terms of antitumor angiogenesis, the promotion of tumor vascular regression and normalization of the remaining blood vessels are equally important. Presently, tumor vessel normalization (TVN) is an emerging antitumor treatment. The present study focused on the normalization of TEC metabolism. The results demonstrated that TECs have a hyperglycolytic metabolism. Parixibox, a cyclooxygenase-2 (COX-2) blocker, successively reduces the expression of vascular endothelial growth factor (VEGF) in the tumor microenvironment. VEGF further influences the expression of 6-Phosphofructo-2-Kinase/Fructose-2,6-Biphosphatase 3, a key glycolysis gene. Pharmacological blockade of COX-2 restored the glucose metabolism level (particularly glycolysis) in TECs, which may be an important basic process in TVN. Therefore, COX-2, which acts on abnormal tumor vessels, is expected to become a novel target for tumor treatment.
\end{abstract}

\section{Introduction}

Normally, solid tumors rely on blood vessels for growth and metastasis (1) because blood vessels can carry oxygen and nutrients to solid tumors and provide a pathway for tumor cell metastasis. Thus, inhibition of blood vessel formation should block the vascular supply and reduce patient mortality and morbidity due to these tumors $(1,2)$. Therapeutic approaches to block the vascular supply have been used in clinical

Correspondence to: Dr Ying Yang, Obstetrics and Gynecology Department, Xinqiao Hospital, Third Military Medical University, 183 Xinqiao Street, Shapingba, Chongqing 400037, P.R. China E-mail: yy630917@126.com

*Contributed equally

Key words: angiogenesis, tumor endothelial cells, tumor vessel normalization, cyclooxygenase-2, parixibox practice; however, these agents may cause hypoxia, which may fuel tumor progression and treatment resistance $(2,3)$. To address this paradox, Jain et al (1), Carretero et al (4) and Peterson et al (5) used the emerging antiangiogenic agents to normalize abnormal tumor vasculature, resulting in the more efficient delivery of drugs and oxygen to targeted cancer cells and the normalization of vascular endothelial cell (EC) connections to reduce tumor cell migration into blood vessels. However the mechanism remains unclear.

Normal and diseased ECs are divided into specialized subtypes, which is the basis for vascular germination. The distal branches of blood vessels play a navigational role but rarely proliferate, while trailing stalk cells proliferate to extend the vascular buds (6). Few reports have demonstrated the metabolic pathways of ECs in vitro, and even fewer reports have studied how EC metabolism regulates angiogenesis in vivo (6). ECs reportedly rely on glycolysis $(7,8)$. One of the rate-limiting checkpoints of glycolytic flux is the conversion of fructose-6-phosphate (F6P) to fructose-1,6 bisphosphate (F1,6P2) through 6-phosphofructo-1-kinase (PFK-1) $(9,10)$. Phosphofructokinase-2/fructose-2,6-bisphosphatase (PFKFB3) enzymes synthesize fructose-2,6-bisphosphate (F2,6P2), an allosteric activator of PFK-1 and the most potent stimulator of glycolysis. Mechanistically, PFKFB3 not only regulates EC proliferation but also controls the formation of filamentous pseudopods and squamous cells and directed migration $(6,11,12)$. Among all PFKFB isozymes, PFKFB3 has the highest kinase activity, which facilitates the production of intracellular F2, 6P2 levels and controls its abundance $(6,9,12)$.

Cyclooxygenase (COX) is an important rate-limiting enzyme in vivo; it is composed of at least two subtypes, COX-1 and COX-2 (13). COX-1 is a normally expressed subtype in the human body (i.e., in the physiological catalysis of arachidonic acid into prostaglandins, which are involved in a variety of important physiological functions). COX-2 is not expressed in most cells; however, it can be induced through a variety of pathological factors, catalyzed by the membrane phospholipid conversion of arachidonic acid produced by prostaglandins and induced through the cascade of amplified inflammatory processes mediated by pathophysiological processes (14). Studies have shown that COX-2 can be induced through inflammation, injury, carcinogens, tumors, and cytokines in a variety of manners to promote tumor progression $(13,15)$. COX inhibitors, also known as non-steroidal anti-inflammatory drugs (NSAIDs), 
reduce the production of inflammatory mediator prostaglandins, produce anti-inflammatory effects, and demonstrate analgesic functions (15). A US epidemiological survey reported that long-term use of low-dose aspirin can reduce the risk of ovarian cancer in women, suggesting that aspirin may prevent ovarian cancer (16); however, aspirin and other COX-1 inhibitors have a wide range of effects. Parecoxib and other selective COX-2 inhibitors not only demonstrate anti-inflammatory and analgesic functions but also prevent the occurrence of tumors; they inhibit tumor development, metastasis and tumor cell proliferation, promote apoptosis, inhibit angiogenesis, and demonstrate significant antitumor effects with few adverse reactions. In addition, the biosafety of these inhibitors is greatly improved relative to traditional treatments $(17,18)$.

Both COX-1 and COX-2 are expressed in ECs (19). However, the effect of COX inhibitors on EC metabolism and tumor vessel normalization (TVN), have been rarely reported. This study aimed to confirm that the inhibitor parecoxib regulates the metabolism of EC glucose through COX-2-VEGF-PFKFB3 signaling pathways, thereby affecting tumor growth.

\section{Materials and methods}

Mice. C57BL/6 mice were obtained from the Animal Institute of the Academy of Medical Science (Beijing, China). The mice were kept under specific pathogen-free conditions at the Animal Center of the Xinqiao Hospital, Third Military Medical University (Chongqing, China). All animal experiments were approved by the Ethics Committee of the Third Military Medical University.

Cell isolation and culture. ID8 cells (mouse ovarian epithelial papillary serous adenocarcinoma) were obtained from the American Type Culture Collection (ATCC). The cells were cultured in DMEM medium (Gibco; Thermo Fisher Scientific, Inc., Waltham, MA, USA) supplemented with $10 \%$ heat-inactivated fetal bovine serum (FBS; Gibco), $2 \mathrm{mM}$ fresh L-glutamine and 100 units/ml penicillin/streptomycin (HyClone; GE Healthcare Life Sciences, Logan, UT, USA) and then maintained in an incubator at $37^{\circ} \mathrm{C}$ with $5 \% \mathrm{CO}_{2}$.

Mouse ECs were isolated from perfused healthy livers or livers infested with ID8 tumors from mice and their wild-type (WT) littermates. Livers were dissected and placed into a $50 \mathrm{ml}$ conical tube containing $5 \mathrm{ml}$ of the digestion buffer. The organs were incubated in a water bath at $37^{\circ} \mathrm{C}$ for approximately $60 \mathrm{~min}$, with regular shaking of the tubes every $10 \mathrm{~min}$. At the end of the digestion process, the tissue was homogeneously dissociated, and the reaction was stopped by adding $10 \mathrm{ml}$ of an isolation buffer containing PBS $+0.1 \%$ EDTA. Subsequently, the cell suspension was filtered through a $100 \mu \mathrm{m}$ cell strainer (Corning Costar, Inc., Corning, NY, USA), and the cells were washed twice with the isolation buffer. Finally, the endothelial cells were isolated by magnetic bead sorting using Dynabeads (CELLection $^{\mathrm{TM}}$ Biotin Binder kit; Thermo Fisher Scientific, Inc.) coated with anti-mouse CD31 (Anti-Mouse CD31 Clone 390; eBioscience; Thermo Fisher Scientific, Inc.), according to the manufacturer's procedure. The purity of the cultured ECs was confirmed by immunofluorescence analysis, showing selective expression of EC-enriched markers.
Tumor challenge experiments. The ID8 cells were cultured until they reached $70 \%$ confluence. Then, WT mice (females, 6-8 weeks old) were subcutaneously injected in the right lateral flank with $5 \times 10^{5}$ ID8 cells in $100 \mu$ l PBS. At the same time, the COX-2 inhibitor group was intraperitoneally injected with $200 \mu \mathrm{l}(250 \mu \mathrm{g} / \mathrm{ml})$ of parixibox, and the control group was injected with $200 \mu \mathrm{l}$ PBS. Tumor sizes were measured using calipers every three days until the mice were sacrificed. The tumor volume was calculated using the following formula: volume $=0.5 \mathrm{x}$ (width) $2 \mathrm{x}$ length.

Immunofluorescence. Immunofluorescence analysis was performed on cell-paved $\mu$-slides. The abovementioned cells were fixed with ice-cold $4 \%$ paraformaldehyde for $20 \mathrm{~min}$ at $37^{\circ} \mathrm{C}$, blocked with normal serum for $20 \mathrm{~min}$ at room temperature and then incubated overnight in the dark at $4^{\circ} \mathrm{C}$ with specific antibodies against mouse monoclonal CD34 (ab 8158; Abcam, Cambridge, UK) and mouse monoclonal factor VIII (9035; Invitrogen ${ }^{\mathrm{TM}}$; Thermo Fisher Scientific, Inc.). After three washes, the slides were stained with Cy3-conjugated anti-mouse antibodies or anti-rabbit antibodies (1:200; Abcam). Nuclei were counterstained with 4,6-diamidino-2-phenylindole (DAPI), and the stained sections were then visualized with a confocal microscope (Olympus Corp., Tokyo, Japan).

Western blot analysis. Proteins of ECs were extracted by RIPA [( $1 \%$ Triton X-100, 0.5\% Na-deoxycholate, $0.1 \%$ sodium dodecyl sulfate (SDS), $20 \mathrm{mmol} / 1$ Tris-HCl (pH 7.4)] with $1 \%$ PMSF (Beyotime Institute of Biotechnology, Haimen, China). Then BCA kit (Beyotime Institute of Biotechnology) was used to examine concentrations of protein. Equal amount of protein samples were resolved on a $10 \%$ SDS-PAGE gel and transferred onto a polyvinylidene difluoride (PVDF) membrane. After blocking with blocking buffer (P0023B; Beyotime Institute of Biotechnology), the membrane was probed with designated primary overnight at $4^{\circ} \mathrm{C}$ : Anti-COX2/Cyclooxygenase 2 antibody (ab62331; Abcam) and mouse anti- $\beta$ Actin antibody (ab8226; Abcam). After washed with $0.1 \%$ TBST, goat anti-rabbit secondary antibody (1:5,000; Beyotime Institute of Biotechnology) and goat anti-mouse secondary antibody (1:5,000; Beyotime Biotechnology) were used respectively, followed by detection of the chemiluminescent signal (Pierce; Thermo Fisher Scientific, Inc.).

Quantitative polymerase chain reaction ( $q P C R$ ). Quantitative PCR was performed to validate the enriched genes observed in microarray experiments. The individual gene expression was analyzed with a PCR kit (Takara, Tokyo, Japan), and carried out in triplicate with an ABI 7300 Prism Sequence Detection System (Applied Biosystems, Foster City, CA, USA). The PCR conditions were as follows: $95^{\circ} \mathrm{C}$ for $30 \mathrm{sec}$, followed by 40 cycles of $95^{\circ} \mathrm{C}$ for $5 \mathrm{sec}, 60^{\circ} \mathrm{C}$ for $34 \mathrm{sec}$, and $72^{\circ} \mathrm{C}$ for $45 \mathrm{sec}$. The relative gene expression levels were calculated using the comparative $\mathrm{Ct}(\Delta \Delta \mathrm{Ct})$ method, with GAPDH as a reference gene. The primers used for PCR.

PFKFB3 forward, CAACTCCCCAACCGTGATTGT and reverse, GAGGTAGCGAGTCAGCTTCTT; COX-2 forward, 


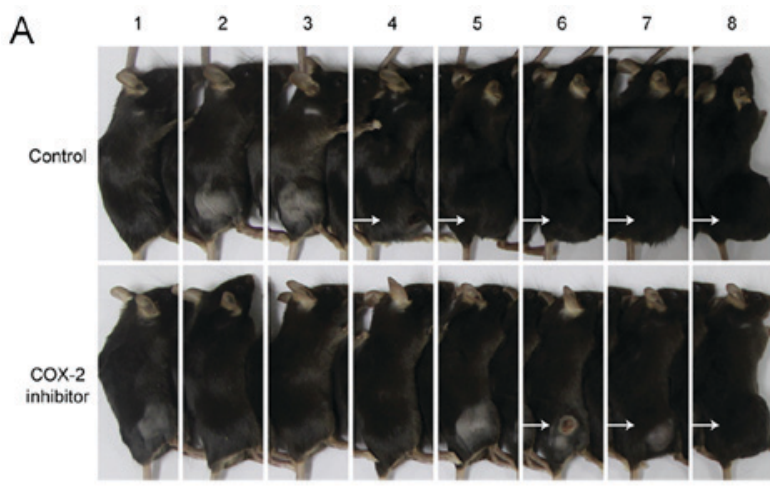

B

\begin{tabular}{|c|c|}
\hline Control & Cox-2 Inhibitor \\
\hline $5 / 8$ & $3 / 8$ \\
\hline
\end{tabular}
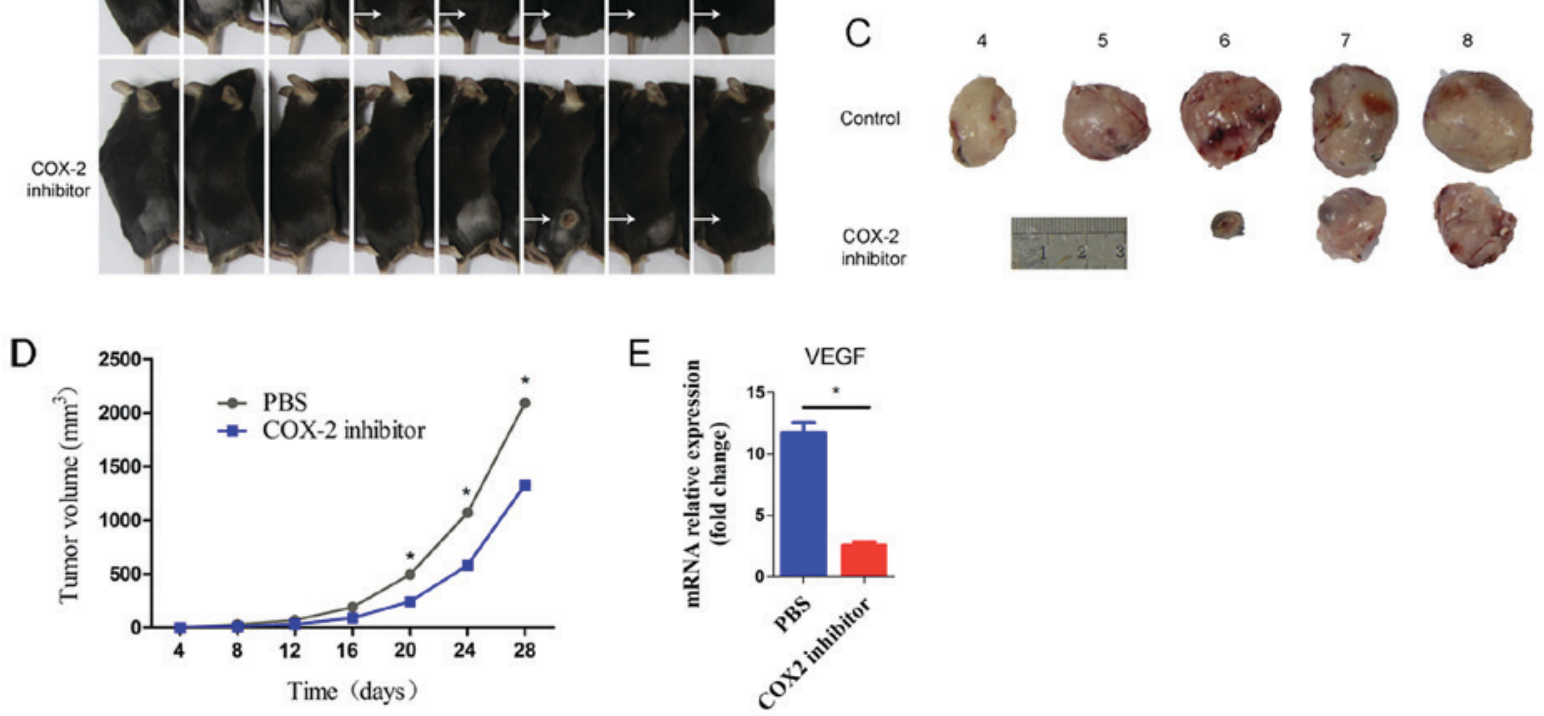

Figure 1. Inhibition of COX-2 by parecoxib impairs tumor progression. A total of $5 \times 10^{5}$ ID8 cells were subcutaneously injected in control or COX-2 inhibitor mice (8 mice per group). (A) Tumors (in vivo) were present when the mice were sacrificed at day 28. (B) Tumor incidence in the two groups. (C) Photographs of the representative tumors in control and COX-2 inhibitor mice are presented. (D) The tumor size was measured, and tumor volumes vs. time are shown. (E) Implanted tumors were harvested from the control and COX-2 inhibitor mice bearing ID8 tumors for 28 days. The expression of VEGF was measured by qRT-PCR. The data shown are representative of three independent experiments. Data represent the means \pm SEM. "P $<0.05$ (A and E, ANOVA followed by Sidak's test for multiple comparisons; B and C, two-tailed unpaired Student's t-test) vs. the indicated comparisons. COX-2, cyclooxygenase-2; VEGF, vascular endothelial growth factor.

CTGCCGTCCGATTGAGACC and reverse, CCCCTCCTT GTACCACTGTC; GAPDH forward, AGGTCGGTGTGA ACGGATTTG and reverse, TGTAGACCATGTAGTTGA GGTCA

Statistical analysis. Statistical analysis data are expressed as mean \pm SEM. Significance was assessed by two-tailed unpaired Student's t-test or other statistical method indicated in the test. $\mathrm{P}<0.05, \mathrm{P}<0.01$ and $\mathrm{P}<0.001$ were considered to indicate a statistically significant difference. Statistical calculations were performed using GraphPad Prism 7.0 (GraphPad Software, Inc., La Jolla, CA, USA). All of the experiments were independently repeated at least 3 times.

\section{Results}

Parecoxib may inhibit tumor progression by downregulating $V E G F$. Previous studies have demonstrated growing evidence for the involvement of COX-2-derived mediators in angiogenic processes. Further demonstrating this role, NSAIDs that selectively block COX-2 activity have both anti-angiogenic and anti-carcinogenic actions $(20,21)$. To investigate whether COX-2 affects the formation and progression of tumors, we used a tumor prevention model to confirm the effect of a COX-2 inhibitor. We subcutaneously injected ID8 cells into WT mice and divided them into two groups. Compared to the control group, parecoxib completely inhibited tumor progression (Fig. 1A). The tumor incidence was $5 / 8$ in the control group and $3 / 8$ in the COX-2 inhibitor group (Fig. 1B). As shown in the figure, the volumes of excised tumors at termination (day 28) were markedly smaller in the COX-2 inhibitor mice than in the control mice (Fig. 1C). Additionally, the tumor growth rate in the COX-2 inhibitor mice was slower than that in the control mice (Fig. 1D). The above data strongly suggest that parecoxib may inhibit tumor formation and progression. Moreover, we found that the VEGF level in the COX-2 inhibitor group was significantly lower than that in the control group (Fig. 1E). The above results are consistent with previously reported results $(22,23)$. These findings imply that the COX-2 inhibitor parecoxib regulates VEGF expression in tumors, which then affects tumor progression. Considering the relationship between VEGF and tumor angiogenesis, we aimed to further clarify the regulation of tumor COX-2/VEGF on tumor vascular ECs, particularly on glucose metabolism.

Expression of COX-2 in ovarian serous cystadenocarcinoma $(O V)$ correlates with VEGF and PFKFB3 expression. To gain more insight into the role of COX-2 in tumor glucose metabolism, we analyzed RNA-seq expression data from The Cancer Genome Atlas (TCGA; cancergenome.nih.gov). Pearson correlation analyses were utilized to examine the correlations. The correlation analyses of COX-2 (Symbol Report: PTGS2) with several genes related to glucose metabolism are shown in Fig. 2. Most analyses demonstrated weak correlations; however, COX-2 and VEGF demonstrated a strong correlation ( $\mathrm{R}=0.41$ ) (Fig. 2A). The same result was observed between VEGF and PFKFB3 (R=0.47, Fig. 2H). PFKFB3 acts as a glycolytic activator, and it is the rate-limiting enzyme in 

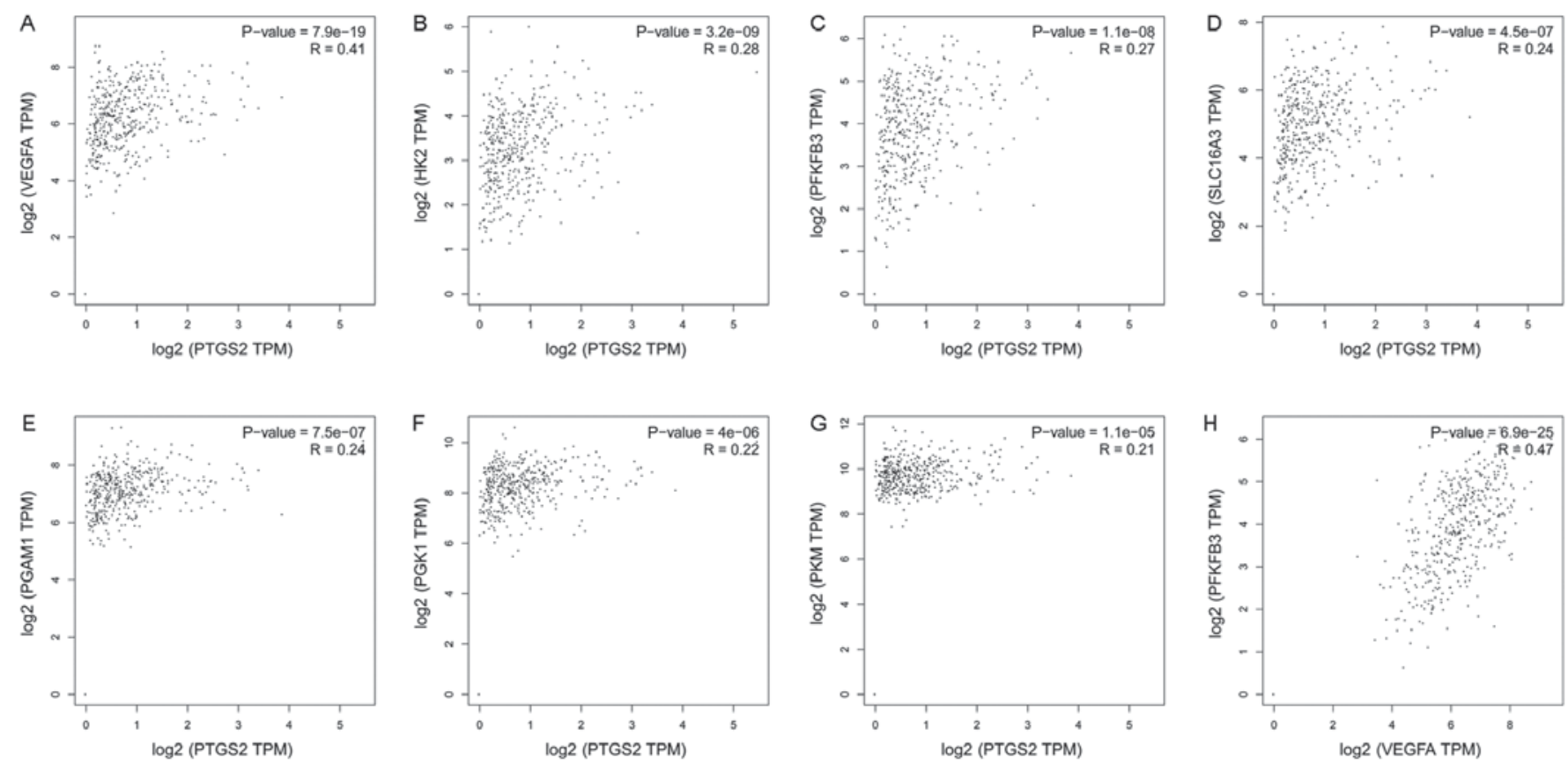

Figure 2. Correlation analysis of glucose metabolism-related genes. We analyzed RNA-seq expression data from TCGA. Pearson's correlation analyses were utilized to examine the correlations; we used the non-log scale for calculations and the log-scale axis for visualization. The following correlations are shown: (A) PTGS2 and VEGFA, (B) PTGS2 and HK2, (C) PTGS2 and PFKFB3, (D) PTGS2 and SLC16A3, (E) PTGS2 and PGAM1, (F) PTGS2 and PGK1, (G) PTGS2 and PKM, (H) VEGFA and PFKFB3. TCGA, The Cancer Genome Atlas; VEGF, vascular endothelial growth factor; PFKFB3, fructose-2,6-bisphosphatase.

glycolysis. The above results suggest that the expression of COX-2 in tumors correlates with VEGF and PFKFB3 expression. However, these results are related to the entire tumor, and the role in ECs is unknown.

Characterization of glucose metabolism in normal and tumor $E C s$. To further understand the mechanistic relationship among COX-2, VEGF and PFKFB3 in ECs, we isolated tumor ECs (TECs) and characterized their metabolic profiles. We injected ID8 cells in the portal vein of WT mice to induce tumor growth in the liver. After 15 days, we isolated ECs from the tumor-infested livers and normal endothelial cells (NECs) from the livers of healthy mice (as the control). First, we identified the TECs and NECs through fluorescence. ECs can be stained to determine whether they are factor VIII (green) positive and CD34 (red) positive; the merged picture in Fig. 3A shows the cytoplasm (yellow) and nucleus (blue). Subsequently, through qRT-PCR, we examined the mRNA expression levels of VEGF and PFKFB3 in NECs and TECs. We noticed obviously increased mRNA expression in TECs compared to NECs (Fig. 3B, C). Meanwhile, we found that the protein levels of PFKFB3 were upregulated (Fig. 3C). We performed glycolysis experiments to determine whether the metabolic activity was greater in TECs or NECs. Our results show that glucose consumption and lactate excretion in the medium were increased in TECs, which suggested hyperglycolysis (Fig. 3D, E). We also noticed an increased glycolytic flux in TECs compared to NECs (Fig. 3F).

COX-2 inhibition induces glucose metabolism normalization in TECs. Because glycolysis activity is greater in TECs than NECs, COX-2, the glycolytic activator PFKFB3 and glycolysis may be attractive targets in TECs. To investigate whether the TEC phenotype could be directly regulated by COX-2, we isolated ECs from the tumor-infested livers and NECs from the livers of healthy mice (as controls); the TECs were incubated with parecoxib $(10 \mu \mathrm{g} / \mathrm{ml})$. We observed that VEGF and PFKFB3 mRNA expression was obviously increased in TECs compared to NECs, while in TECs exposed to parecoxib, the mRNA expression was obviously decreased compared to that in the non-exposed TECs and NECs (Fig. 4A, B). In addition, the protein levels of PFKFB3 were downregulated in the TEC+inhibitor group compared to the TECs and NECs (Fig. 4B). To determine which group demonstrated greater glucose metabolism, we also performed a glycolysis experiment. Similar to the gene results, our results showed that the glucose consumption and lactate excretion of the TECs were increased in the medium, which suggested that TECs were hyperglycolytic, and the glycolysis levels of the COX-2 inhibitor-induced TECs tended to be normal (Fig. 4C, D).

COX-2 inhibition regulates glycolysis pathways in TECs. ECs rely on glycolysis instead of oxidative metabolism for adenosine triphosphate (ATP) production $(11,24)$. The switch from a quiescent to an angiogenic phenotype, which occurs in cancer, is metabolically demanding and mediated by adaptations in EC metabolism. Lactate dehydrogenase B (LDH-B) is upregulated in the tumor endothelium, and VEGF signaling increases glycolytic flux by inducing GLUT1 and the glycolytic enzyme PFKFB3 $(11,25)$. PFKFB3 catalyzes the synthesis of fructose-2,6-bisphosphate (F2,6P2), which is an allosteric activator of PFK-1. In our study, we used a COX-2 inhibitor to treat TECs; it first inhibited the function of COX-2, resulting in decreased VEGF production and PFKFB3 expression. The process of TEC glycolysis is affected in this manner, and the products of glycolysis and ATP are altered, resulting in a change in the biological function of TECs (Fig. 5). 
A

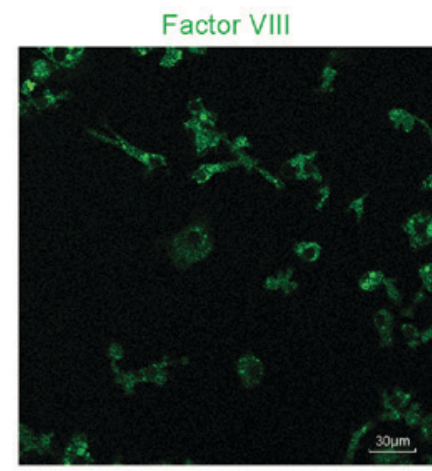

\section{B}

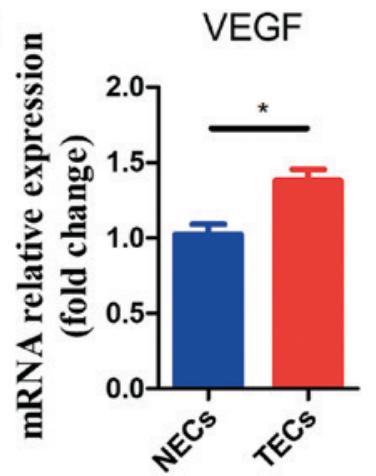

D

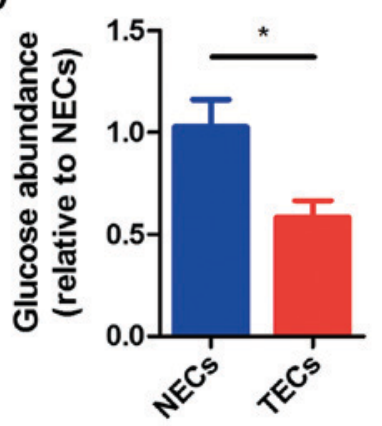

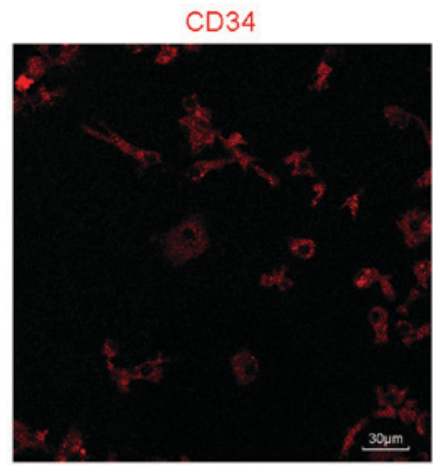

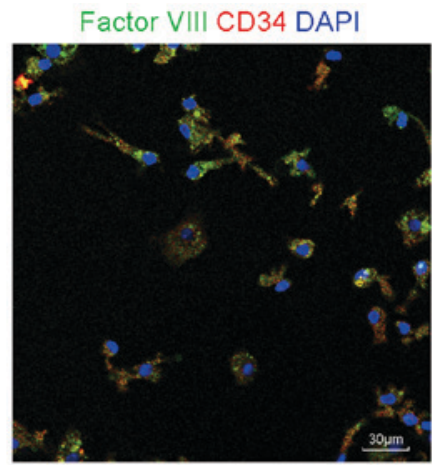

C

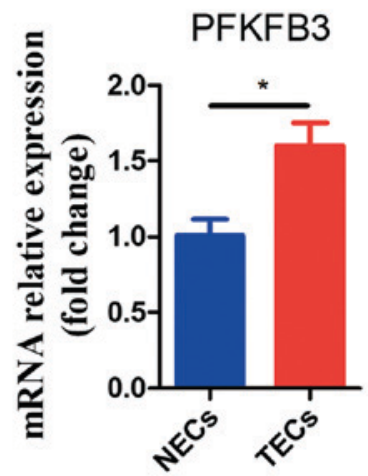

E

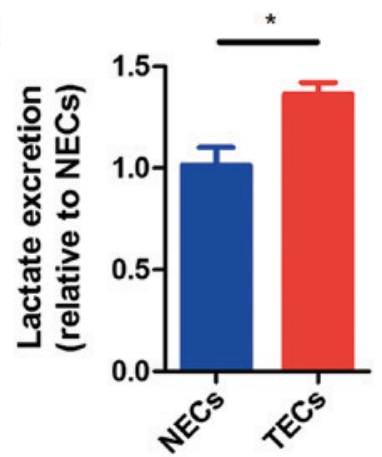

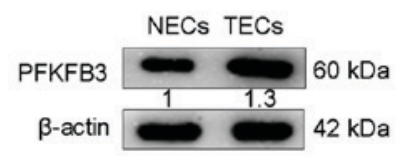

F

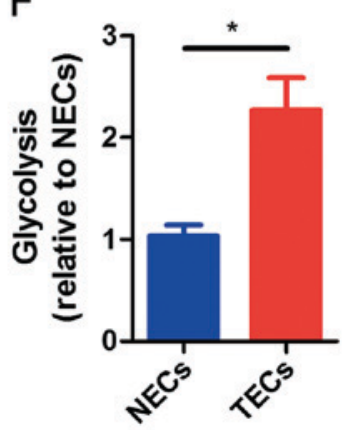

Figure 3. Characterization of normal and tumor endothelial cells. (A) In the immunofluorescence assay, ECs were stained for factor VIII (green) and CD34 (red). The merged image shows yellow and blue areas. (B) qRT-PCR of mRNA expression levels of VEGF in NECs and TECs. The values are expressed relative to NECs (n=3). (C) qRT-PCR of mRNA expression and WB of protein expression levels of PFKFB3 in NECs and TECs. The values are expressed relative to NECs ( $n=3)$. (D) Glucose levels in the medium of TECs relative to NECs ( $n=3)$. (E) Lactate levels in the medium of TECs relative to NECs (n=3). (F) Glycolytic flux in TECs relative to NECs $(n=3)$. All data are shown as the means \pm SEM. ${ }^{*} \mathrm{P}<0.05$. ECs, endothelial cells; TECs, tumor endothelial cells; VEGF, vascular endothelial growth factor; PFKFB3, fructose-2,6-bisphosphatase; NECs, normal endothelial cells.

\section{Discussion}

ECs are arranged in the lumen of the blood vessel and are, therefore, exposed to high concentrations of oxygen in the blood. Thus, we suspected that these cells are better adapted to oxidative metabolism. However, vascular germination is not inhibited by mitochondrial respiration (6). In fact, ECs were found to be highly glycolytic, resulting in up to an $85 \%$ increase in ATP production. Even if glycolysis produces only two ATP molecules per molecule of glucose, the glycolytic EC provides a wide variety of advantages over the production of 34 molecules of ATP produced by the normal oxidative metabolism of glucose (6). For example, anaerobic glucose metabolism allows ECs to vascularize blood vessels without hypoxia. In addition, glycolysis can produce more ATP molecules than oxidants in a shorter time span than the normal metabolic pathway, thereby rapidly providing ECs with the necessary energy to germinate, form new blood vessels and rapidly evolve to restore the oxygen supply of the surrounding tissue (26). In addition to the glycolytic metabolites in the bypass pathway, the production of biomass requires macromolecules and can also further regulate the function of redox homeostasis, which is conducive to rapid vascular germination.

VEGF increases the expression of PFKFB3 through glycolysis. Reducing glycolysis by PFKFB3 gene inactivation in ECs reduces the migration of tip cells and the proliferation of stem cells to prevent vascular germination (6). In in vitro models, silencing of PFKFB3 reduced EC movement, lamellipodia/filopodia formation and directed migration and damaged EC spheroids (27). In addition, EC PFKFB3-deficient mice showed impaired vascular growth and branch formation in the neonatal retinal vasculature due to reduced stem cell proliferation and damaged filamentous pseudopodia. Further analysis showed that PFKFB3 was co-localized with F-actin 
A

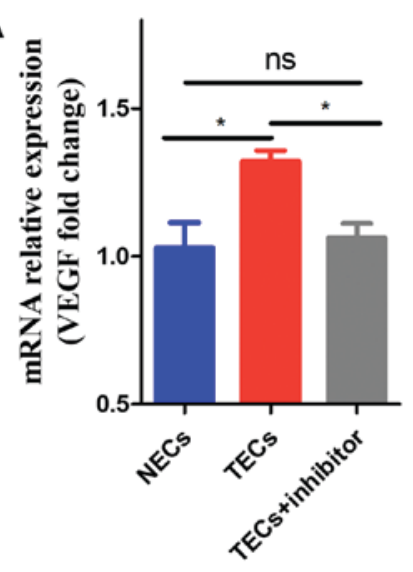

C

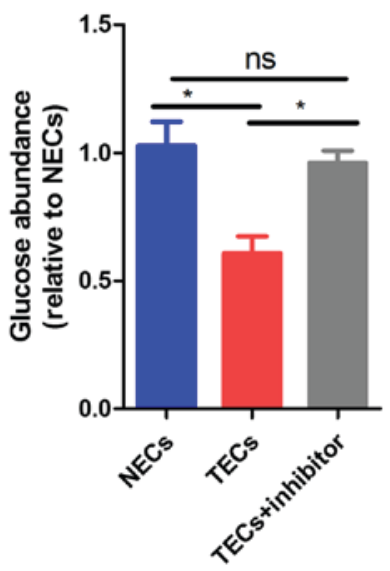

B

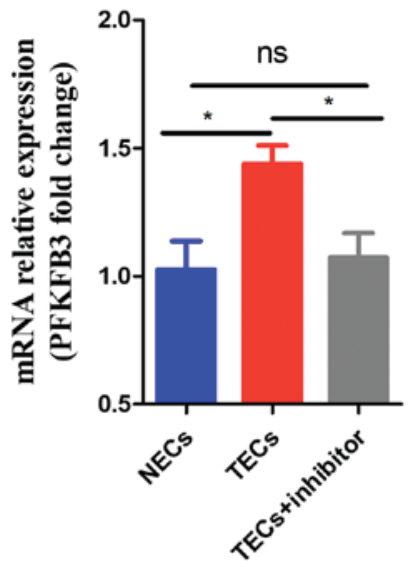

D

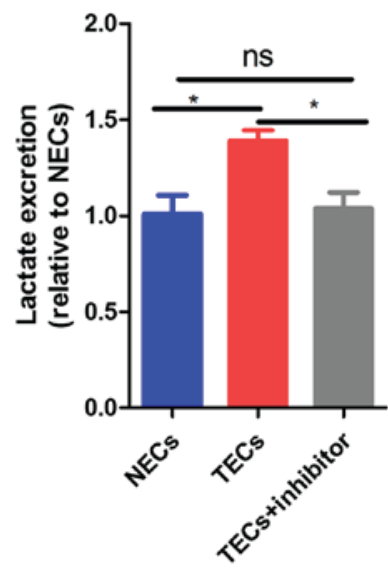

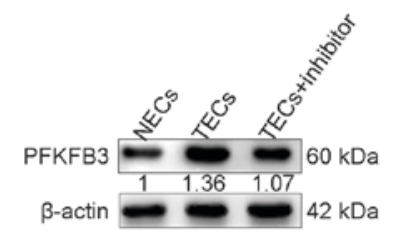

阝-actin , 
not alter the relative expression level of stem cells, suggesting that the metabolic changes alone, particularly PFKFB3-driven glycolysis, were sufficient to promote activity and vascular germination.

EC dysfunction, which occurs when ECs are no longer able to perform their physiological functions, may also contribute to cardiovascular disease and diabetes (25). Thus, inhibition of pathological vascular germination or prevention or reduction of EC dysfunction is valuable for a wide variety of diseases. For the treatment of overreaction, the most well-known and, to date, the only clinically approved antiangiogenic strategy relies on growth factor signaling (primarily VEGF signaling). This method is inadequate for cancer treatment because of endogenous intolerance or resistance to the secretion of angiogenic factors due to an increased compensatory mechanism, which results in only limited benefits. The finding that EC metabolism codetermines blood vessel formation may provide a novel opportunity for antiangiogenic treatment based on an entirely different mechanism. Indeed, PFKFB3 represents a target that interferes with glycolysis and may counteract abnormal and excessive formation of blood vessels in disease (28).

TVN is emerging as an anti-cancer treatment. Previous TVN strategies have focused on targeting angiogenic signals; however, targeting EC glucose metabolism as a method of promoting TVN is still rare. Our data showed that COX-2 inhibition reduces TEC glycolysis as follows: (1) TECs are hyperglycolytic, and pharmacological blockade of COX-2 in TECs induces glycolysis normalization. (2) In vivo, the pharmacological blockade of COX-2 impairs tumor progression.

COX-2 expression is associated with VEGF expression in a variety of malignant tumors. Overexpression of COX-2 in tumor tissue can promote angiogenesis, and VEGF may play a mediating role. In Hodgkin's lymphoma, COX-2 overexpression was found to be associated with angiogenesis, which induced Bcl-2 and VEGF expression. COX-2 induces VEGF expression in the following manner: (1) PGE2 upregulates the expression of VEGF in gastric cancer cells by transactivating the EGFR-MAPK signaling pathway, which is one of the basic mechanisms of COX-2 that promotes tumor angiogenesis (13); (2) COX-2 promotes tumor angiogenesis through the PGE2/HIF-1 $\alpha /$ VEGF signaling pathway (14); (3) COX-2, when overexpressed in vivo, can activate the epidermal growth factor receptor (EGFR) and increase the production of cyclic adenosine monophosphate (cAMP) by producing PGE2 and activating the cAMP response element binding protein (CREB).

COX inhibitors have become an important target for the treatment of malignant tumors in recent years, and they have been shown to have a significant inhibitory effect on some malignant biological behaviors of tumor cells. Masferrer and others have found that COX inhibitors can reduce the expression of angiogenic factors and the downstream biological effects of COX-2. Our results showed that pharmacological blockade of COX-2 in TECs can reduce VEGF expression in the tumor microenvironment, and VEGF can further influence the expression of PFKFB3, a key enzyme gene of glycolysis. Pharmacological blockade of COX-2 in TECs restored glycolysis in tumor vascular ECs, which may be an important basis for the normalization of tumor blood vessels.
COX-2 inhibitors have been shown to play an important role in the treatment of a variety of tumors as well as vascular abnormalities. Based on our findings, we have reason to believe that COX-2 and TVN may become new targets for tumor prevention and treatment.

\section{References}

1. Jain RK: Normalization of tumor vasculature: An emerging concept in antiangiogenic therapy. Science 307: 58-62, 2005.

2. Potente M, Gerhardt $\mathrm{H}$ and Carmeliet P: Basic and therapeutic aspects of angiogenesis. Cell 146: 873-887, 2011.

3. Jain RK: Antiangiogenesis strategies revisited: From starving tumors to alleviating hypoxia. Cancer Cell 26: 605-622, 2014.

4. Carretero R, Sektioglu IM, Garbi N, Salgado OC, Beckhove P and Hämmerling GJ: Eosinophils orchestrate cancer rejection by normalizing tumor vessels and enhancing infiltration of CD8(+) T cells. Nat Immunol 16: 609-617, 2015.

5. Peterson TE, Kirkpatrick ND, Huang Y, Farrar CT, Marijt KA, Kloepper J, Datta M, Amoozgar Z, Seano G, Jung K, et al: Dual inhibition of Ang-2 and VEGF receptors normalizes tumor vasculature and prolongs survival in glioblastoma by altering macrophages. Proc Natl Acad Sci USA 113: 4470-4475, 2016.

6. De Bock K, Georgiadou M, Schoors S, Kuchnio A, Wong BW, Cantelmo AR, Quaegebeur A, Ghesquière B, Cauwenberghs S, Eelen G, et al: Role of PFKFB3-driven glycolysis in vessel sprouting. Cell 154: 651-663, 2013.

7. Polet $\mathrm{F}$ and Feron $\mathrm{O}$ : Endothelial cell metabolism and tumour angiogenesis: Glucose and glutamine as essential fuels and lactate as the driving force. J Intern Med 273: 156-165, 2013.

8. Cruys B, Wong BW, Kuchnio A, Verdegem D, Cantelmo AR, Conradi LC, Vandekeere S, Bouché A, Cornelissen I, Vinckier S, et al: Glycolytic regulation of cell rearrangement in angiogenesis. Nat Commun 7: 12240, 2016.

9. Xu Y, An X, Guo X, Habtetsion TG, Wang Y, Xu X, Kandala S, Li Q, Li H, Zhang C, et al: Endothelial PFKFB3 plays a critical role in angiogenesis. Arterioscler Thromb Vasc Biol 34: 1231-1239, 2014.

10. Cantelmo AR, Conradi LC, Brajic A, Goveia J, Kalucka J, Pircher A, Chaturvedi P, Hol J, Thienpont B, Teuwen LA, et al: Inhibition of the glycolytic activator PFKFB3 in endothelium induces tumor vessel normalization, impairs metastasis and improves chemotherapy. Cancer Cell 30: 968-985, 2016.

11. De Bock K, Georgiadou M and Carmeliet P: Role of endothelial cell metabolism in vessel sprouting. Cell Metab 18: 634-647, 2013.

12. Schoors S, De Bock K, Cantelmo AR, Georgiadou M, Ghesquière B, Cauwenberghs S, Kuchnio A, Wong BW, Quaegebeur A, Goveia J, et al: Partial and transient reduction of glycolysis by PFKFB3 blockade reduces pathological angiogenesis. Cell Metab 19: 37-48, 2014.

13. Di Francesco L, Dovizio M, Trenti A, Marcantoni E, Moore A, O'Gaora P, McCarthy C, Tacconelli S, Bruno A, Alberti S, et al: Dysregulated post-transcriptional control of COX-2 gene expression in gestational diabetic endothelial cells. Br J Pharmacol: Jul 3, 2015 (Epub ahead of print).

14. Leahy KM, Ornberg RL, Wang Y, Zweifel BS, Koki AT and Masferrer JL: Cyclooxygenase- 2 inhibition by celecoxib reduces proliferation and induces apoptosis in angiogenic endothelial cells in vivo. Cancer Res 62: 625-631,2002.

15. Sui W, Zhang Y, Wang Z, Wang Z, Jia Q, Wu L and Zhang W: Antitumor effect of a selective COX-2 inhibitor, celecoxib, may be attributed to angiogenesis inhibition through modulating the PTEN/PI3K/Akt/HIF-1 pathway in an $\mathrm{H}_{22}$ murine hepatocarcinoma model. Oncol Rep 31: 2252-2260, 2014

16. Gaul C: Aspirin for migraine in pregnancy. This recommendation seems questionable. MMW Fortschr Med 155: 17, 2013 (In German).

17. Salvado MD, Alfranca A, Haeggström JZ and Redondo JM: Prostanoids in tumor angiogenesis: Therapeutic intervention beyond COX-2. Trends Mol Med 18: 233-243, 2012.

18. Bijman MN, Hermelink CA, van Berkel MP, Laan AC, Janmaat ML, Peters GJ and Boven E: Interaction between celecoxib and docetaxel or cisplatin in human cell lines of ovarian cancer and colon cancer is independent of COX-2 expression levels. Biochem Pharmacol 75: 427-437, 2008. 
19. Di Francesco L, Totani L, Dovizio M, Piccoli A, Di Francesco A Salvatore T, Pandolfi A, Evangelista V, Dercho RA, Seta F and Patrignani P: Induction of prostacyclin by steady laminar shear stress suppresses tumor necrosis factor-alpha biosynthesis via heme oxygenase-1 in human endothelial cells. Circ Res 104: 506-513, 2009

20. de Groot DJ, de Vries EG, Groen HJ and de Jong S: Non-steroidal anti-inflammatory drugs to potentiate chemotherapy effects: From lab to clinic. Crit Rev Oncol Hematol 61: 52-69, 2007.

21. Burleigh ME, Babaev VR, Yancey PG, Major AS, McCaleb JL, Oates JA, Morrow JD, Fazio S and Linton MF: Cyclooxygenase-2 promotes early atherosclerotic lesion formation in ApoE-deficient and C57BL/6 mice. J Mol Cell Cardiol 39: 443-452, 2005.

22. Yoshida S, Amano H, Hayashi I, Kitasato H, Kamata M, Inukai M, Yoshimura $\mathrm{H}$ and Majima M: COX-2/VEGF-dependent facilitation of tumor-associated angiogenesis and tumor growth in vivo. Lab Invest 83: 1385-1394, 2003.

23. Wu G, Luo J, Rana JS, Laham R, Sellke FW and Li J: Involvement of COX-2 in VEGF-induced angiogenesis via P38 and JNK pathways in vascular endothelial cells. Cardiovasc Res 69: 512-519, 2006.
24. Vandekeere S, Dewerchin M and Carmeliet P: Angiogenesis revisited: An overlooked role of endothelial cell metabolism in vessel sprouting. Microcirculation 22: 509-517, 2015.

25. Goveia J, Stapor P and Carmeliet P: Principles of targeting endothelial cell metabolism to treat angiogenesis and endothelial cell dysfunction in disease. EMBO Mol Med 6: 1105-1120, 2014.

26. Eelen G, de Zeeuw P, Simons M and Carmeliet P: Endothelial cell metabolism in normal and diseased vasculature. Circ Res 116: 1231-1244, 2015.

27. Sun CM, Xiong DB, Yan Y, Geng J, Liu M and Yao XD: Genetic alteration in phosphofructokinase family promotes growth of muscle-invasive bladder cancer. Int J Biol Markers 31: e286-e293, 2016.

28. Schoors S, Bruning U, Missiaen R, Queiroz KC, Borgers G, Elia I, Zecchin A, Cantelmo AR, Christen S, Goveia J, et al: Fatty acid carbon is essential for dNTP synthesis in endothelial cells. Nature 520: 192-197, 2015. International (CC BY-NC-ND 4.0) License. 